\title{
QUASICIRCLES AND HARMONIC MEASURE
}

\author{
JAN G. KRZYZ̈
}

\section{Introduction}

It is well-known that a quasiconformal (abbreviated: qc) self-mapping of a Jordan domain $G$ has a homeomorphic extension on the closure $\bar{G}$ which generates a sense-preserving homeomorphism of the boundary $\partial G$ onto itself. In this way a $K$-qc automorphism of $G$ induces an automorphism of $\partial G$.

The boundary correspondence problem for qc mappings, i.e., the problem of characterizing the induced automorphisms of $\partial G$, can be reduced after suitable conformal mappings to the case $G=U:=\{z: \operatorname{Im} z>0\}$ and admits in this particular case a very simple and elegant solution. Suppose that an automorphism of $\boldsymbol{R}=\partial U$ is determined by a continuous, strictly increasing function $h$ which satisfies $h(-\infty)=-\infty, h(+\infty)=+\infty$. According to Beurling and Ahlfors ([3]), $h$ can be extended to an automorphism of $\bar{U}$ which is $K$-qc in $U$, if and only if there exists a constant $M=M(K)$ such that

$$
M^{-1} \leqq \frac{h(t+d)-h(t)}{h(t)-h(t-d)}:=h(t, d) \leqq M ; \quad t, d \in R, \quad d \neq 0 .
$$

The function $h$ satisfying the condition (1.1) (referred to as the $M$-condition) is said to be quasisymmetric on $\boldsymbol{R}$.

The necessity of the $M$-condition (see e.g. [6]) was obtained by considering the modules of quadrilaterals arising from $U$ by distinguishing four points on the boundary. There are, however, two one-parameter configurations involving points on the boundary, the quadrilateral and a punctured Jordan domain with two distinguished points on the boundary curve, cf. [2], p. 70. Therefore we may expect that the boundary correspondence generated by qc automorphisms with one fixed interior point may be described in terms of the characteristic conformal invariant of the latter configuration, the harmonic measure. In fact, we were able to obtain Theorems 1, 2 as the solution to this problem. The necessary and sufficient condition (2.1) characterizing the boundary correspondence is conformally invariant and at the same time as simple as the original $M$-condition (1.1). Another advantage is that no special boundary point is distinguished in (2.1). Actually, the assumption of the existence of an interior fixed point does not mean any loss of generality. In the 
case of the upper half-plane $U$ we can make of any point $z_{0} \in U$ a fixed point of the qc extension $f$ without changing the boundary correspondence. This can be done by composing $f$ with a suitable affine self-mapping $w \mapsto a w+b \bar{w}, a+b=1$, $|a|>|b|$, of $U$, that keeps the points of $\partial U$ unchanged.

As an application we establish a new characterization of quasicircles in terms of harmonic measure (Theorem 3), which adds to the seventeen characterizations already known as listed in [4], p. 27.

The results mentioned above were presented at the Rolf Nevanlinna Colloquium in Zürich on June 13, 1985.

\section{Functions quasisymmetric on $T$}

Let $\boldsymbol{T}$ denote the boundary of the unit disk $\boldsymbol{D}$ and let $\tilde{h}$ be an automorphism of $T$, i.e., a sense-preserving homeomorphism of $T$ onto itself. If $\alpha$ is an open subarc of $T$, then $|\alpha|$ will stand for the harmonic measure $\omega(0, \alpha ; D)$.

Definition 1 . We say that $\tilde{h}$ is quasisymmetric on $T$ if and only if there exists a constant $M$ such that for any pair $\alpha_{1}, \alpha_{2}$ of disjoint adjacent open subarcs of $T$ with equal harmonic measures $\left|\alpha_{1}\right|=\left|\alpha_{2}\right|$ we have

$$
\left|\tilde{h}\left(\alpha_{1}\right)\right| /\left|\tilde{h}\left(\alpha_{2}\right)\right| \leqq M .
$$

In what follows (2.1) will be called the $M$-condition on $T$. Obviously $M \geqq 1$. Suppose $\alpha(t)$ is a variable arc on $T$ of constant harmonic measure $|\alpha(t)|=1 / 2$ determined by its end-point $\exp (2 \pi i t), t \in \boldsymbol{R}$. If we rotate $\alpha(t)$ by the angle $\pi$ from the initial position corresponding to $t=t_{0}$, then the function $t \mapsto 1 / 2-|\tilde{h}(\alpha(t))|$ takes for $t=t_{0}$ and $t=t_{0}+1 / 2$ opposite values and therefore it vanishes for some $\tau_{0} \in\left[t_{0} ; t_{0}+1 / 2\right]$. This means that $\left|\alpha\left(\tau_{0}\right)\right|=\left|\tilde{h}\left(\alpha\left(\tau_{0}\right)\right)\right|=1 / 2$ and, consequently, after suitable rotations, the automorphism $\tilde{h}$ can be normalized so as to satisfy

$$
\tilde{h}(1)=1, \quad \tilde{h}(-1)=-1 .
$$

The automorphism $\sigma=\tilde{h}(\tau)$ induces an automorphism $s=h(t)$ of the interval $[0 ; 1)$, where $s=(2 \pi)^{-1} \arg \sigma, t=(2 \pi)^{-1} \arg \tau$ and arg ranges over $[0 ; 2 \pi)$. Thus with the corresponding branch of logarithm we have

$$
s=(2 \pi i)^{-1} \log \tilde{h}(\exp (2 \pi i t))=(2 \pi)^{-1} \arg \tilde{h}(\tau):=h(t) .
$$

Since $\lim h(t)=1$ as $t \rightarrow 1$, we may consider $h$ an automorphism of $I=[0 ; 1]$ normalized by the conditions

$$
h(0)=0, \quad h(1 / 2)=1 / 2, \quad h(1)=1 .
$$

The automorphism $h$ can be extended to an automorphism of $\boldsymbol{R}$ by putting

$$
h(t+1)=h(t)+1 \text {. }
$$


Then evidently (2.3) holds for all $t \in \boldsymbol{R}$ with a suitable continuous branch of logarithm, and the condition (2.1) takes the form

$$
M^{-1} \leqq \frac{h(t+d)-h(t)}{h(t)-h(t-d)} \leqq M ; \quad t \in I, \quad 0<|d| \leqq 1 / 2 .
$$

We now prove that the restrictions on $t, d$ in (2.6) imply those in (1.1), i.e., we have

Lemma 1. If $h$ is a strictly increasing function on $\boldsymbol{R}$ which satisfies (2.4)-(2.6), then the conditions (1.1) and (2.6) are equivalent.

In other words, the $M$-condition on $T$ for $\tilde{h}$ is equivalent to the $M$-condition on $\boldsymbol{R}$ for the associated function $h$ with the same value of $M$.

Proof. It is sufficient to prove that (2.6) implies (1.1). Note first that the function $t \mapsto h(t, d)$ is periodic with period 1 and hence the assumption $t \in \boldsymbol{R}$ may be replaced by $t \in I$. In view of the identity $h(t, d)=1 / h(t,-d)$ we may assume that $d>0$. If $d=n$ is a positive integer, then obviously $h(t, d)=1$ for all $t \in \boldsymbol{R}$ so that (1.1) certainly holds. Otherwise $d=n+\delta$, where $0<|\delta| \leqq 1 / 2$ and $n$ is a non-negative integer. If $0<\delta \leqq 1 / 2$, then

$$
h(t, d)=\frac{n+h(t+\delta)-h(t)}{n+h(t)-h(t-\delta)}=\frac{x+a}{x+b},
$$

where $x=n \geqq 0$ and $a, b>0$. The function $x \mapsto(x+a) /(x+b)$ is for $a \neq b$ strictly monotonic in $[0 ;+\infty)$ and has extreme values $1, a / b=h(t, \delta)$, where $t \in I, 0<\delta \leqq 1 / 2$. Hence the bounds for $h(t, d)$ and $h(t, \delta)$ are the same in this case. Suppose next that $d=n+\delta>0$ and $-1 / 2 \leqq \delta<0$. Then $n \geqq 1$ and

$$
h(t, d)=[n-(h(t)-h(t+\delta))] /[n-(h(t-\delta)-h(t))]=(x-a) /(x-b),
$$

where $x \geqq 1,0<a, b<1, a+b=h(t-\delta)-h(t+\delta) \leqq 1$. The function $x \mapsto(x-a) /(x-b)$ is again strictly monotonic in $[1 ;+\infty)$ for $a \neq b$ and has extreme values 1 , $(1-a) /(1-b)$. If $a<b$, then the upper bound $(1-a) /(1-b) \leqq b / a$ because of $a+b \leqq 1$. If $b<a$, then the lower bound $(1-a) /(1-b) \geqq b / a$ by the same argument. Thus the bounds for $h(t, \delta), t \in I, 0<|\delta| \leqq 1 / 2$, are the same as the bounds for $h(t, d)$, where $t, d \in \boldsymbol{R}, d \neq 0$, and this proves our lemma.

Corollary 1. Lemma 1 is an improvement on Theorem 5 [5], under an additional and quite natural assumption.

Suppose now that $\lambda(K)$ is the distortion function

$$
\lambda(K)=\left[\mu^{-1}(\pi K / 2)\right]^{-2}-1,
$$

where $\mu(r)$ is the module of the ring domain $D \backslash[0 ; r]$. Then, as an immediate consequence of Lemma 1 and Theorem 6.2 [6] we obtain

Theorem 1. The boundary values $\tilde{h}$ of a $K$-qc self-mapping $H$ of $\boldsymbol{D}$ with $H(0)=0$ satisfy the inequality

$$
\left|\tilde{h}\left(\alpha_{1}\right)\right| /\left|\tilde{h}\left(\alpha_{2}\right)\right| \leqq \lambda(K)
$$


for any pair of disjoint adjacent open subarcs $\alpha_{1}, \alpha_{2}$ of $T$ with equal harmonic measures $\left|\alpha_{1}\right|=\left|\alpha_{2}\right|$.

Proof. After suitable rotations $h$ will satisfy (2.2) and the relations $z=(2 \pi i)^{-1} \log \tau, w=(2 \pi i)^{-1} \log \sigma$, where $\sigma=H(\tau)$ and $\log 1=0$, generate a $K$-qc self-mapping $\Phi$ of $U$ given by

$$
w=(2 \pi i)^{-1} \log H(\exp (2 \pi i z)):=\Phi(z) .
$$

Therefore the restriction $\Phi \mid \boldsymbol{R}=h$ satisfies the $M$-condition on $\boldsymbol{R}$ with $M=\lambda(K)$, cf. [6], Theorem 6.2. However, $h$ is associated with $\tilde{h}$ by (2.3) and by Lemma $1, \tilde{h}$ must satisfy the $M$-condition on $T$, i.e. (2.8), with the same value of $M$. This completes the proof.

We can also easily prove the sufficiency of the $M$-condition. We have

Theorem 2. Let $\tilde{h}$ be an automorphism of $T$ which satisfies the inequality (2.1) for all pairs $\alpha_{1}, \alpha_{2}$ of disjoint adjacent open subarcs of $T$ with equal harmonic measures $\left|\alpha_{1}\right|=\left|\alpha_{2}\right|$. Then there exists a quasiconformal self-mapping $H$ of the unit disk $\boldsymbol{D}$ satisfying $H(0)=0$ which has the boundary values $H \mid T=\tilde{h}$ and the maximal dilatation not greater than $K=K(M)$.

Proof. Given $\tilde{h}$ satisfying (2.1), perform first suitable rotations so as to obtain the normalization (2.2) and then consider the function $h$ representing the automorphism of $\boldsymbol{R}$ satisfying (2.3)-(2.5). As shown in Lemma $1, h$ satisfies the $M$-condition on $\boldsymbol{R}$ with the same value of $M$ as in (2.1). According to Beurling and Ahlfors (see e.g. [1], p. 69), the function $w=\Phi(z)=u(x, y)+i v(x, y), z=x+i y$, where

$$
u(x, y)=(2 y)^{-1} \int_{-y}^{y} h(x+t) d t, \quad v(x, y)=(2 y)^{-1} \int_{-y}^{y}[h(x+t)-h(x-t)] d t,
$$

represents an explicit $K$-qc automorphism of $U$ with boundary values $h$ and the maximal dilatation $K$ depending only on $M$. We may take e.g. $K(M)=8 M$, cf. [7]. It follows from (2.5) and (2.10) that

$$
u(x+1, y)=1+u(x, y), \quad v(x+1, y)=v(x, y) .
$$

This property enables us to construct the self-mapping $\sigma=H(\tau)$ of $D \backslash\{0\}$ defined as follows:

$$
\sigma=\exp [2 \pi i \Phi((1 /(2 \pi i)) \log \tau)]:=H(\tau) .
$$

Since the singularity at 0 is removable, $H$ can be extended to the whole disk $\boldsymbol{D}$ as a $K$-qc mapping with boundary values $\tilde{h}$ on $T$. This completes the proof. 


\section{Quasicircles and harmonic measure}

As an application of the results given in the preceding section we shall obtain the following characterization of quasicircles in terms of harmonic measure.

Theorem 3. Let $L$ be a Jordan curve in the extended plane $\hat{C}$ and let $G, G^{*}$ be its complementary domains. The curve $L$ is a quasicircle if and only if there exist two points $z^{\prime} \in G, z^{\prime \prime} \in G^{*}$ and a constant $M \geqq 1$ such that for any two adjacent disjoint open subarcs $\alpha_{1}, \alpha_{2}$ of $L$ such that

$$
\omega\left(z^{\prime}, \alpha_{1} ; G\right)=\omega\left(z^{\prime}, \alpha_{2} ; G\right)
$$

we have

$$
\omega\left(z^{\prime \prime}, \alpha_{1} ; G^{*}\right) / \omega\left(z^{\prime \prime}, \alpha_{2} ; G^{*}\right) \leqq M .
$$

Proof. Necessity. Since $L$ is a quasicircle, there exists a $K$-qc self-mapping $\Psi$ of the extended plane such that $L=\Psi(T)$. Suppose that $G=\Psi(D), G^{*}=\Psi\left(D^{*}\right)$, where $D^{*}=\{z:|z|>1\}$, and take $z^{\prime}=\Psi(0), z^{\prime \prime}=\Psi(\infty)$. If $\sigma(w)=1 / \bar{w}$, then the mapping

$$
\varphi=\Psi \circ \sigma \circ \Psi^{-1}
$$

is a qc reflection in $L$ and $z^{\prime \prime}=\varphi\left(z^{\prime}\right)$. Let $g, g^{*}$ be conformal mappings of $G$ and $G^{*}$ onto $D$ and $D^{*}$, respectively, so chosen that $g\left(z^{\prime}\right)=0, g^{*}\left(z^{\prime \prime}\right)=\infty$. We now define the qc self-mapping $H$ of $D, H(0)=0$, in the following way:

$$
H=\sigma \circ g^{*} \circ \varphi \circ g^{-1} \text {. }
$$

Consider the restriction $H \mid T=\tilde{h}$ and two adjacent disjoint open subarcs $\beta_{1}$, $\beta_{2}$ of $T$ with equal harmonic measures $\left|\beta_{1}\right|=\left|\beta_{2}\right|$. For their image arcs under $\tilde{h}$ we have

$$
\left|\tilde{h}\left(\beta_{1}\right)\right| /\left|\tilde{h}\left(\beta_{2}\right)\right| \leqq M
$$

by (2.8). Due to conformal invariance of harmonic measure we have $\omega\left(z^{\prime}, \alpha_{1} ; G\right)=$ $\omega\left(z^{\prime}, \alpha_{2} ; G\right)$, where $\alpha_{k}=g^{-1}\left(\beta_{k}\right), k=1,2$. The reflection $\varphi$ does not change the arcs $\alpha_{k}$ and carries $z^{\prime}$ into $z^{\prime \prime}$. Since the conformal mapping $g^{*}$ does not change harmonic measure, we have $\omega\left(z^{\prime \prime}, \alpha_{k} ; G^{*}\right)=\omega\left(\infty, \tilde{h}\left(\beta_{k}\right) ; D^{*}\right)=\left|\tilde{h}\left(\beta_{k}\right)\right|$ so that (3.5) implies (3.2).

Sufficiency. Let $L$ be a Jordan curve such that the inequality (3.2) holds for any pair of disjoint adjacent open subarcs $\alpha_{1}, \alpha_{2}$ of $L$ that satisfy (3.1). Conformal mappings $g: G \rightarrow D, g^{*}: G^{*} \rightarrow D^{*}$ such that $g\left(z^{\prime}\right)=0, g^{*}\left(z^{\prime \prime}\right)=\infty$, induce an automorphism $\tilde{h}=g^{*} \circ g^{-1}$ of $T$. After suitable rotations $\tilde{h}$ will satisfy (2.2). Due to (3.1), (3.2) and the conformal invariance of harmonic measure, we have for any pair of disjoint adjacent open subarcs $\beta_{1}, \beta_{2}$ of $T$ with $\left|\beta_{1}\right|=\left|\beta_{2}\right|$ the inequality $\left|\tilde{h}\left(\beta_{1}\right)\right| /\left|\tilde{h}\left(\beta_{2}\right)\right| \leqq M$. Therefore, by Theorem 2 there exists a qc self-mapping $H$ of 
$D, H(0)=0$, with boundary values $\tilde{h}$. Consider now the composite mapping

$$
\varphi=\left(g^{*}\right)^{-1} \circ \sigma \circ H \circ g \text {. }
$$

Obviously $\varphi$ is a sense-reversing qc mapping of $G$ onto $G^{*}$. Moreover, $g^{*} \circ g^{-1}=$ $\tilde{h}=H \mid T$ so that $\varphi=\left(g^{*}\right)^{-1} \circ \sigma \circ\left(g^{*} \circ g^{-1}\right) \circ g=$ id on $L$. This means that $\varphi$ is a qc reflection in $L$ and, consequently, $L$ is a quasicircle, as a Jordan curve admitting qc reflection, cf. [6], p. 99. This completes the proof.

\section{References}

[1] AHLFors, L. V.: Lectures on quasiconformal mappings. - D. Van Nostrand Company, Inc., Princeton, N. J.-Toronto-New York-London, 1966.

[2] Ahlfors, L. V.: Conformal invariants - Topics in geometric function theory. - McGraw-Hill Book Company, New York et al., 1973.

[3] Beurling, A., and L. V. Ahlfors: The boundary correspondence under quasiconformal mappings. - Acta Math. 96, 1956, 125-142.

[4] Gehring, F. W.: Characteristic properties of quasidisks. Séminaire de Mathématiques Supérieures, 84. - Presses de l'Université de Montréal, Montréal, Que., 1982.

[5] Kelingos, J. A.: Boundary correspondence under quasiconformal mappings. - Michigan Math. J. 13, 1966, 235-249.

[6] Lehto, O., and K. I. ViRTANEN: Quasiconformal mappings in the plane. - Springer-Verlag, Berlin-Heidelberg-New York, 1973.

[7] REED, T. J.: Quasiconformal mappings with given boundary values. - Duke Math. J. 33, 1966, $459-464$.

Uniwersytet Marii Curie-Sklodowskiej

Instytut Matematyki

P1. M. Curie-Skłodowskiej 1

PL 20-031 Lublin

Poland

Received 19 June 1985 